Article

\title{
A Novel Approach for Synthesis of Layered Manganese Oxide Nanocomposites through a Delamination/Reassembling Process
}

\author{
Zong-huai LIU, Xiaojing YANG, Yoji MAKITA, and Kenta OOI \\ National Institute of Advanced Industrial Science and Technology, 2217-14, Hayashi-cho, Takamatsu, Kagawa, \\ 761-0395 Japan
}

(Manuscript submitted February 14; 2003; accepted March 31, 2003)

\begin{abstract}
Layered manganese oxide nanocomposites are important inorganic materials due to their unique adsorptive, catalytic and electrochemical properties. However, it is difficult to synthesize layered manganese oxide nanocomposites with bulky guest ions or molecules in the interlayer by a conventional intercalation reaction due to the high charge density in the interlayer for layered manganese oxides. This paper reports a novel approach for synthesis of layered manganese oxide nanocomposites through a delamination/reassembling process. This new technology is effective and can be used widely for various kinds of guest ions or molecules to develop novel selective adsorbents, catalysts, or cathode materials for lithium batteries.
\end{abstract}

\section{Introduction}

There has been considerable research activity in the synthesis of inorganic-organic nanocomposite materials because these materials have proved to exhibit a variety of unique properties due to the synergies of the inorganic and organic components.[1] Layered nanocomposites are materials generally comprised of organic molecules or ions incorporated between sheets of an inorganic host. The host layered compounds include transition metal halides, clays, oxides, and layered metal phosphorus chalcogenides, etc.[2]

Layered manganese oxides are important two-dimensional layered inorganic materials due to their unique adsorptive, catalytic, and electrochemical properties.[3] Some organic alkylammonium ions have been intercalated into the interlayer by an ion-exchange reaction, accompanied by the expansion of the interlayer. The expanded layered manganese nanocomposites can be used as molecular sieves or precursors to synthesize porous manganese oxides.[4-6] However, layered manganese oxides have a higher charge density in the interlayer than the other layered materials, making the synthesis of layered manganese oxide nanocomposites with organic molecules or bulky guest ions in the interlayer by a conventional intercalation reaction difficult. For the first time, we found that the layered manganese oxide can be delaminated into its nanosheets by the intercalation of organic tetramethylammonium ions into the interlayer, followed by water washing.[7] The delaminated manganese oxides have a higher degree of freedom, and the organic molecules or bulky guest ions can be easily adsorbed onto their nanosheet surface. Layered manganese oxide nanocomposites of ultimate two-dimensionality with thickness in the subnano- to nanometer range, good crystallinity, and novel physical 
properties can thus be fabricated. This paper reports the novel approach for synthesis of layered manganese oxide nanocomposites through the delamination/reassembling process.

\section{Experimental}

\subsection{Materials}

The host material, proton-type birnessite manganese oxide $(\operatorname{BirMO}(\mathrm{H}))$, was prepared by the acid treatment of highly crystallized sodium-type birnessite (BirMO(Na)) in a $0.1 \mathrm{M} \mathrm{HCl}$ solution.[8] The acid-treated sample has a chemical formula of $\mathrm{H}_{3.49} \mathrm{Na}_{0.21} \mathrm{Mn}_{12} \mathrm{O}_{23} \cdot 9.5 \mathrm{H}_{2} \mathrm{O}$ and cation exchange capacity of 2.70 $\mathrm{mmol} / \mathrm{g}$.

Delamination of $\operatorname{BirMO}(\mathrm{H})$ was carried out by the method we have described previously.[7] $\mathrm{BirMO}(\mathrm{H})$ $(0.5 \mathrm{~g})$ was treated in a $0.35 \mathrm{M}$ tetramethylammonium hydroxide (TMAOH) solution $\left(125 \mathrm{~cm}^{3}\right)$ in a $250 \mathrm{~cm}^{3}$ glass bottle while stirring for 7 days at $25^{\circ} \mathrm{C}$. The amount of TMAOH added was 25 -fold of the exchangeable capacity for $\operatorname{BirMO}(\mathrm{H})$. The suspension was centrifuged and colloidal sediment was washed 4 times with 100 $\mathrm{cm}^{3}$ of distilled water, after which exfoliated BirMO slurry was obtained.

Tetramethylammonium hydroxide (TMAOH) and poly(diallydimethylammonium) (PDDA) chloride $\left(M_{\mathrm{w}} \approx 300,000\right)$ were purchased from Wako Pure Chemical Co. 3-Aminopropyltrimethoxysilane $\left(\left(\mathrm{CH}_{3} \mathrm{O}\right)_{3} \mathrm{Si}\left(\mathrm{CH}_{2}\right)_{3} \mathrm{NH}_{2}\right)$ (AMPS) and Stearyltrimethylammonium (STMA) bromide $\left(\mathrm{CH}_{3}\left(\mathrm{CH}_{2}\right)_{17} \mathrm{~N}\left(\mathrm{CH}_{3}\right)_{3} \cdot \mathrm{Br}\right)$ were obtained from Kasei Chemical Co.

\subsection{Preparation}

The delaminated BirMO slurry was poured into an aqueous solution of TMAOH, an aqueous solution of PDDA chloride, a mixed solution of 1-hexanol and AMPS, or an aqueous solution of STMA bromide, respectively. The ratios of added organic ions or molecules $(\mathrm{mmol})$ to birnessite $(\mathrm{H})(\mathrm{mmol})$ were adjusted depending on their properties and structures. Each mixture was maintained overnight while stirring at room temperature. The product was collected by centrifugation, washed with distilled water, and then dried for 2 days at room temperature. Organic ion or molecule-intercalated layered manganese oxide nanocomposites were obtained, and they are hereafter abbreviated as TMAMO, PDDAMO, AMPSMO, and STMAMO for TMA-, PDDA-, AMPS-, and STMA-intercalated materials, respectively.

\section{Results and Discussion}

\subsection{Delamination of BirMO(H)}

Starting $\operatorname{BirMO}(\mathrm{H})$ has a layered structure with a basal spacing of $0.73 \mathrm{~nm}$ (Fig. 1a). BirMO(H) was soaked in a TMAOH solution while stirring for 7 days at room temperature, after which a colloidal suspension was obtained. The suspension was filtered, and the colloidal sediment was subjected to XRD measurement in its wet state. The wet-state XRD patterns show that the basal spacing of $\operatorname{BirMO}(\mathrm{H})$ changes from $0.73 \mathrm{~nm}$ to $1.56 \mathrm{~nm}$ (Fig. 1b). This indicates that the intercalation of $\mathrm{TMA}^{+}$ions causes a swelling along the layers. The basal spacing of $1.56 \mathrm{~nm}$ corresponds to a single layer of $\mathrm{TMA}^{+}$ions with two water layers between manganese oxide lamellae. Chemical analysis results for the intercalated sample after air-drying show that the intercalated amount of $\mathrm{TMA}^{+}$ions reached $85 \%$ of the ion exchange capacity of $\mathrm{BirMO}(\mathrm{H})$. The amounts of $\mathrm{OH}^{-}$consumed in the supernatant solution were nearly equal to $\mathrm{TMA}^{+}$uptakes, indicating that the intercalation reaction progresses by an ion-exchange mechanism. 
The colloidal sediment was washed 4 times with distilled water. The wet-state XRD pattern of the washed sample gives no clear peaks but only an amorphous halo (Fig. 1c), in striking contrast to that of the unwashed sample. The halo can be interpreted as scattering from the nanosheets which are aggregated irregularly in the slurry. This indicates that water washing causes the delamination of stacked manganese oxide plates into the individual primary plates. Water washing causes a simultaneous decrease of both the sheet charge density and the interlayer salt concentration. The decrease of the sheet charge density results in a decrease of the electrostatic attractive force between interlayer cations and anionic sheets. The decrease of the ion concentration of the interlayer solution strengthens the repulsive force between the manganese oxide sheets, owing to the decrease of the shielding effect provided by the interlayer ions. Thus the decreases in ion concentration and surface charge density result in the delamination of a stacked manganese oxide.

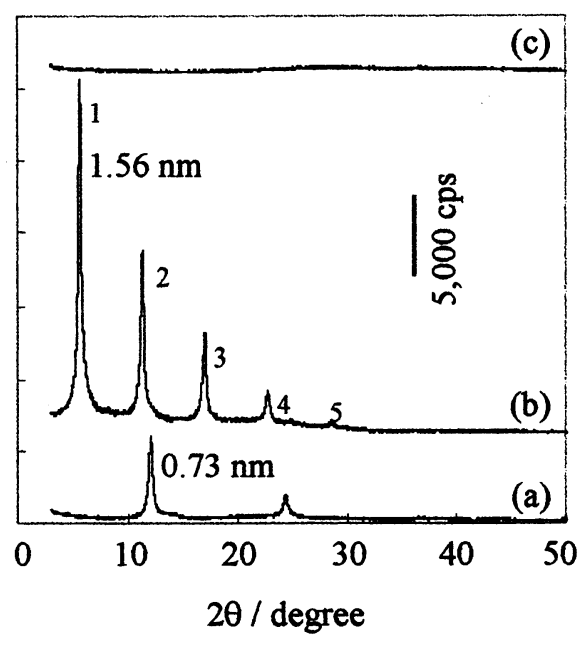

Fig. 1 XRD patterns of (a) BirMO (H), (b) $\mathrm{TMA}^{+}$-intercalated sample in wet state, and (c) delaminated BirMO slurry.

\subsection{Reassembling process of manganese oxide nanosheets}

The delaminated BirMO slurry was added to solutions containing different guest ion or molecule solutions at room temperature and stirred for a day. After stirring, the precipitates were washed and dried at room temperature for 2 days. A series of new layered phases with different basal spacings were obtained (Fig. 2). This shows that the reassembling of delaminated sheets progresses merely by mixing with different guest ions

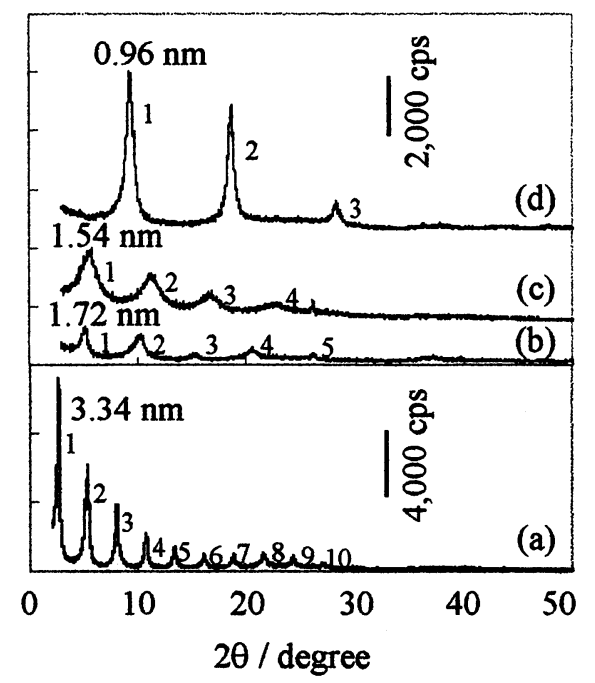

Fig. 2 XRD patterns of layered manganese oxide nanocomposites: (a) STMAMO, (b) AMPSMO, (c) PDDAMO, and (d) TMAMO. or molecules followed by air-drying. The presence of sharp diffraction lines suggests that the manganese oxide sheets are stacked in an ordered manner. The differences in expansion of the layer relate to differences in the size of intercalated guest ions or molecules in the interlayer. The basal spacing and the stacked order of layered manganese oxide nanocomposites depend on the size and properties of the guest ions or molecules.

The basal spacing increases with an increase in the size of intercalated guest ions. Considering the thickness $(0.45 \mathrm{~nm})$ of the manganese oxide sheet, the net interlayer expansion can be evaluated. For TMAMO, the net interlayer expansion was $0.51 \mathrm{~nm}$ due to a relatively small ion radius for $\mathrm{TMA}^{+}$ions $(0.44 \mathrm{~nm})$, corresponding to the intercalation of unhydrated $\mathrm{TMA}^{+}$(Fig. 2d). PDDAMO had a net interlayer expansion of $1.09 \mathrm{~nm}$, which corresponds to two molecular layers of PDDA chains since the effective thickness of PDDA is known to be about $0.5 \mathrm{~nm}$ (Fig. 2c). AMPSMO was synthesized in a mixed solution of 1-hexanol and AMPS. The 1-hexanol solution is suitable for the suppression of rapid hydrolysis/precipitation of AMPS during the reaction with the slurry. The methoxy groups of AMPS are mostly 
hydrolyzed while the propylamine groups remain unchanged, being chemically bonded to $\mathrm{Si}$ atoms, or $(\mathrm{OH})_{3} \mathrm{Si}\left(\mathrm{CH}_{2}\right)_{3} \mathrm{NH}_{2}$. While some of the hydroxyl groups may be condensed to form a polymerized silica material. Hydrolyzed organic silicates $\left((\mathrm{OH})_{3} \mathrm{Si}\left(\mathrm{CH}_{2}\right)_{3} \mathrm{NH}_{2}\right)$ intercalate into the interlayer, resulting in an interlayer spacing of $1.27 \mathrm{~nm}$ (Fig. 2b). STMAMO has a large interlayer distance $(2.89 \mathrm{~nm})$ due to the large size of STMA ions $(2.65 \mathrm{~nm})$. Sharp diffraction lines up to the tenth order indicate a highly ordered stacked structure (Fig. 2a).

These results show that the reassembling reaction progresses soft involving the intercalation reaction. The delamination/reassembling method is a simple and effective method for the preparation of layered manganese oxide nanocomposites with large ions or molecules in the interlayer.

\subsection{Formation process for layered manganese oxide nanocomposites}

A formation process for layered manganese oxide nanocomposites can be written as shown in Fig. 3 . Starting $\operatorname{BirMO}(\mathrm{H})$ contains one molecular layer of water between the manganese oxide sheets with a basal spacing of $0.73 \mathrm{~nm}$. BirMO(H) is soaked in TMAOH solution for 7 days, during which the intercalation of $\mathrm{TMA}^{+}$ions takes place, accompanied by a swelling of the interlayer up to a basal spacing of $1.56 \mathrm{~nm}$. When the swollen material is washed with distilled water 4 times, a suspension of exfoliated manganese oxide colloidal is obtained. When the colloidal suspension is added to a solution containing different guest ions or molecules, they are adsorbed on the surface of the exfoliated manganese oxide sheets. The sheets

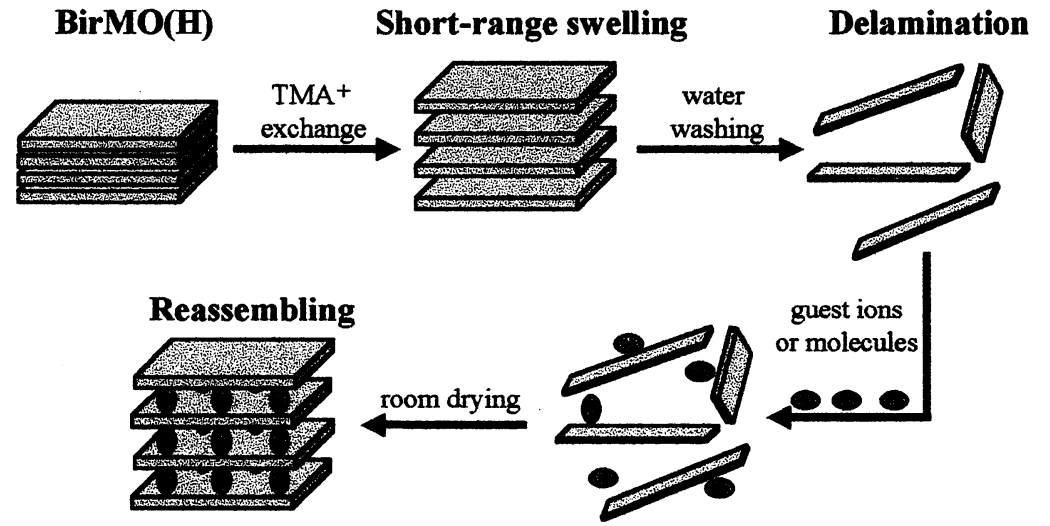

Fig. 3 Formation process of layered manganese oxide nanocomposites are reassembled by room drying, with guest ions or molecules taking up position in the interlayers of the manganese oxide sheets. Thus, layered manganese oxide nanocomposites with different basal spacings can be synthesized. This new method is promising for the synthesis of many kinds of functional manganese oxide nanocomposites since it can be used for widely different kinds of guests involving bulky ions or molecules.

\section{References}

1) C. Sanchez, G. J. De A. A. Soler-Illia, F. Ribot, T. Lalot, C. R. Mayer, and V. Cabuil, Chem. Mater., 13, 3061-3083 (2001).

2) N. Sukpirom and M. M. Lerner, Chem. Mater., 13, 2179-2185 (2001).

3) Q. Feng, H. Kanoh, and K. Ooi, J. Mater. Chem., 9, 319-333 (1999).

4) Q. Gao, O. Giraldo, W. Tong, and S. L. Suib, Chem. Mater. 13, 778-786 (2001).

5) Z.-H. Liu, K. Ooi, H. Kanoh, W. Tang, X. Yang, and T. Tomida, Chem. Mater., 13, 473-478 (2001).

6) T. Ressler, S. L. Brock, J. Wong, and S. L. Suib, J. Phys. Chem. B, 103, 6407-6420 (1999).

7) Z.-H. Liu, K. Ooi, H. Kanoh, W. Tang, and T. Tomida, Langmuir, 16, 4154-4164 (2000).

8) Q. Feng, E.-H. Sun, K. Yamagisawa, N. Yamasaki, J. Ceram. Soc. Jpn. 105, 564-569 (1997). 